\title{
Comparison of Curative Effects between Minimally Invasive Percutaneous Lag Screw Internal Fixation and Reconstruction Plate for Unstable Pelvic Fractures
}

\author{
Jifeng Fang1, Hui Dong2 and Zihui Li ${ }^{3}$ \\ ${ }^{1}$ Department of Orthopaedics, Second People's Hospital of Liaocheng, Linqing City, Shandong Province, China \\ ${ }^{2}$ Department of Hand and Foot Microsurgery, The Sixth Affiliated Hospital of Xinjiang Medical University, Urumqi, Xinjiang, China \\ ${ }^{3}$ Department of Orthopedics, Altay District People's Hospital, Xinjiang, China
}

\begin{abstract}
Objective: To compare the clinical efficacy of minimally invasive percutaneous lag screw internal fixation and reconstruction plate in the treatment of unstable pelvic fractures.

Study Design: An experimental study.

Place and Duration of Study: The Department of Orthopaedics, Second People's Hospital of Liaocheng, Linqing City, China, from January 2016 to March 2018.

Methodology: A total of 130 patients with unstable pelvic fracture were randomly divided into group $A$ and group $B$, with 65 cases in each group. Group A was treated with minimally invasive percutaneous lag screw internal fixation, and group B was treated with reconstruction plate. Clinical efficacy of two groups after treatment was evaluated. Serum alkaline phosphatase (ALP), tumor necrosis factor- $\alpha$ (TNF- $\alpha$ ), C-reactive protein (CRP), stress hormones cortisol (Cor), angiotensin II (Ang II), and norepinephrine (NE) contents were compared.

Results: After treatment, the excellent and good rate in group A was higher than that in group $B(p=0.005)$. At 7 days after the operation, serum ALP level in group A was higher than that in group $B(p<0.001)$, serum TNF- $\alpha, C R P$, Cor, Ang II, and $\mathrm{NE}$ levels were lower in group $A$ than those in group $B$ (all $p<0.001$ ).

Conclusion: Compared with reconstruction plate, minimally invasive percutaneous lag screw internal fixation has better clinical efficacy in patients with unstable pelvic fractures; meanwhile, it can effectively increase serum ALP level, reduce the levels of inflammatory cytokines TNF- $\alpha$ and CRP, and effectively reduce surgical trauma as well as postoperative body stress response.
\end{abstract}

Key Words: Hollow lag screw, Minimally invasive surgery, Reconstruction plate, Unstable pelvic fracture, Stress hormone.

How to cite this article: Fang J, Dong H, Li Z. Comparison of curative effects between minimally invasive percutaneous lag screw internal fixation and reconstruction plate for unstable pelvic fractures. J Coll Physicians Surg Pak 2020; 30(1):28-32.

\section{INTRODUCTION}

Unstable pelvic fractures are clinically common types of fractures. They are mainly caused by traffic accidents and falling. Patients with unstable pelvic fractures are often associated with ankle dislocation and peripheral bone structural damage.1,2 Without timely treatment, severe cases are prone to compound injuries and hemorrhagic shock, thereby endangering patients' lives. ${ }^{3,4}$ Currently, clinical treatment of pelvic fractures includes conservative or surgical treatment. Conservative treatment mainly adopts pelvic suspension, cast immobilisation, traction, etc. However, its efficacy is poor, the treatment is painful, and the prognosis is not stable.5,6 Recently, minimally invasive percutaneous lag screw internal fixation and reconstruction plate surgical methods have received increasing attention. Pilling et al.

Correspondence to: Dr. Zihui Li,Department of Orthopaedics, Altay District People's Hospital, Xinjiang, China-836500

E-mail:lozy36@163.com

Received: May 14, 2018; Revised: September 27, 2019;

Accepted: November 26, 2019 found that minimally invasive fracture treatment could ensure stable fixation of the fracture with a minimum of osteosynthesis material, and reduce the operative time. ${ }^{7}$ Zhang et al. revealed that minimally invasive percutaneous lag screws are feasible for the treatment of acetabular anterior column fractures. ${ }^{8}$ Tempelaere et al. demonstrated that minimally invasive percutaneous fixation of vertically unstable pelvic fractures can produce good functional and radiological outcomes. ${ }^{9}$ According to a study, reconstruction plate internal fixation is a safe and effective way to treat unstable pelvic posterior ring fractures. ${ }^{10} \mathrm{How}-$ ever, there are very few studies comparing the minimally invasive percutaneous lag screw internal fixation with reconstruction plate internal fixation in the treatment of unstable pelvic fractures.

The objective of this study was to compare the clinical efficacy between minimally invasive percutaneous lag screw internal fixation and reconstruction plate for unstable pelvic fractures, in order to provide more reference for the clinical treatment of unstable pelvic fractures. 


\section{METHODOLOGY}

This experimental study was conducted at the Department of Orthopaedics, Second People's Hospital of Liaocheng, Linqing City, China, from January 2016 to March 2018. The study was approved by the Hospital Ethics and Research Committee. The inclusion criteria comprised those who were diagnosed with unstable pelvic fractures, and had closed pelvic fractures, confirmed by pelvic radiography and $\mathrm{CT}$, without any other serious liver and kidney diseases, and mentally normal. Exclusive criteria were who suffered severe tissue or organ organic diseases, and did not meet surgical indications, with mental disorders, unwilling to receive treatment, unwilling to cooperate in the study, and who were transferred or died in mid-course. A total of 130 patients with unstable pelvic fractures were randomly divided into group $A$ and group $B$, with 65 cases in each group, according to random number table method. All patients were applied with traction on the femoral condyle. When the patients showed stable vital signs and hemodynamic conditions, surgical treatment was conducted after confirming complete or basic reduction by the X-ray or pelvic CT scan and three-dimensional reconstruction. Both groups were given continuous epidural anesthesia or general anesthesia. Anterior ring reduction and internal fixation (pubic ramus or pubic symphysis) were first performed in the pelvis; and then posterior ring reduction and internal fixation were performed.

Group A patients underwent minimally invasive percutaneous lag screw fixation. The anterior pelvic ring was fixed in the supine position with a percutaneous hollow lag screw; and the posterior pelvic ring was fixed with a percutaneous iliac screw in the prone position. The ipsilateral lower limb traction was performed and the C-arm fluoroscopy was used before fixation, including the pelvic inlet, outlet, and normal position. A small incision of about $1 \mathrm{~cm}$ was taken $3-5 \mathrm{~cm}$ at the side of the posterior superior iliac spine; the soft tissue was separated to reach the sacrum directly. The positioning cannula was placed into the incision and reaches the sacrum. At the normal position and the outlet, fluoroscopy cannula was parallel to the upper part of the $\mathrm{S} 1$ and the upper edge of the sacral foramen. From that, tilting forward 20 degrees further and 20 degrees upwards, the screw was slowly inserted with $2 \mathrm{~mm}$ diameter guide pin. Through fluoroscopy, the anteversion angle is controlled to avoid the spinal canal at the entrance and the oblique angle of view at the exit to avoid the sacral nerve hole. The length of the screw is grasped by lateral fluoroscopy of the tibia and the guide pin is passed through the tibia and ankle to the S1 vertebral body. Under normal conditions, the tip of the guide pin was near the midline. In a standard lateral perspective, the tip of guide pin was in the front of the S1 vertebral body, indicating that the guide pin was in the correct position. A $7.3 \mathrm{~mm}$ diameter hollow compression screw was slowly screwed in along the guide pin.

Patients in the group B were treated with reconstruction plate: through incision over the upper level of the pubic symphysis in a supine position, the bilateral pubic body was fully exposed, and the reconstruction plate was placed directly above and/or ahead of the pubis and fixed with screws. A curved incision of about $6 \mathrm{~cm}$ was made along the lateral side of the posterior superior iliac spine on both sides in a prone position. After the skin was cut into the subcutaneous deep fascia, the posterior superior iliac spine was displayed. The gluteal muscle was peeled off outwardly and laterally from the outer plate of the sacrum. The outer plate was exposed, and a reconstruction plate, the length of which can be fixed with 2-3 screws on both sides, was used. Before fixation, one end of the steel plate was pre-curved, and the steel plate was sent to the opposite side through the subcutaneous tunnel from the affected side. The other end of the plate was pre-bent and placed on to the dorsal side of both sides of the humerus. Screws were screwed on the sides of the plate and were able to penetrate the bilateral cortex. Antibiotics were routinely administered to both groups to prevent infection and reduce swelling.

After treatment, the clinical efficacy of two groups of patients was evaluated. The clinical efficacy criteria included excellent, good, moderate and poor. The equal length of limbs after treatment, no rotation of the lower limbs, normal gait, and no pain at the fracture site were expressed as excellent. The length difference of limbs was less than $2 \mathrm{~cm}$, and no rotation of the lower limbs, basically normal gait, and no pain at the fracture site were expressed as good. The length difference of limbs as long as $2-3 \mathrm{~cm}$; rotation of the lower limbs $<15$ degrees, slight limp, and slight pain at the fracture site were expressed as moderate. The length difference of limbs $>4 \mathrm{~cm}$, the rotation of the lower limbs $>15$ degrees, obvious limp, and pain at the fracture site were expressed as poor.

At 7 days after operation, $5 \mathrm{~mL}$ of cubital venous blood was collected from the two groups at a speed of $3000 \mathrm{r} / \mathrm{min}$ and centrifuged for $15 \mathrm{~min}$. The serum was separated and stored in a low temperature cabinet at $-50^{\circ} \mathrm{C}$ for detection of serum ALP, TNF- $\alpha$, CRP and stress hormone markers. Enzyme-linked immunosorbent assay was used to detect serum TNF- $\alpha$ and CRP levels. The patients' serum ALP levels were measured using an automated biochemical analyser. Radioimmunoassay was used to determine the level of stress hormones in the patients' serum, including Cor, Ang II, and NE.

Data were entered and analysed by using SPSS 22.0. Measurement data were calculated as mean $\pm S D$, and independent-sample t-test was used to compare the outcome between two groups. Count data were indicated in $\mathrm{n}(\%)$, and Chi-square test was used to compare the 
Table I: Comparison of serum ALP, TNF- $\alpha$ and CRP levels 7 days after operation in two groups.

\begin{tabular}{|c|c|c|c|c|c|c|c|}
\hline \multirow[t]{2}{*}{ Groups } & \multirow[t]{2}{*}{$\mathrm{n}$} & \multicolumn{2}{|c|}{ ALP (U/L) } & \multicolumn{2}{|c|}{ TNF- $\alpha(\mathrm{ng} / \mathrm{mL})$} & \multicolumn{2}{|c|}{ CRP (mg/L) } \\
\hline & & Mean $\pm S D$ & $p$-value & Mean \pm SD & $p$-value & Mean \pm SD & $p$-value \\
\hline Group B & 65 & $81.74 \pm 6.28$ & $<0.001$ & $1.76 \pm 0.35$ & $<0.001$ & $13.26 \pm 1.85$ & $<0.001$ \\
\hline Group A & 65 & $87.31 \pm 7.55$ & & $1.13 \pm 0.21$ & & $11.14 \pm 1.6$ & \\
\hline
\end{tabular}

Table II: Comparison of serum stress hormone levels 7 days after operation in two groups.

\begin{tabular}{|c|c|c|c|c|c|c|c|}
\hline \multirow[t]{2}{*}{ Groups } & \multirow[t]{2}{*}{$\mathrm{n}$} & \multicolumn{2}{|c|}{ Cor (ng/mL) } & \multicolumn{2}{|c|}{ Ang II (ng/L) } & \multicolumn{2}{|c|}{$\mathrm{NE}(\mathrm{ng} / \mathrm{mL})$} \\
\hline & & Mean \pm SD & $p$-value & Mean \pm SD & $p$-value & Mean \pm SD & $p$-value \\
\hline Group B & 65 & $263.21 \pm 24.83$ & $<0.001$ & $51.53 \pm 5.12$ & $<0.001$ & $0.49 \pm 0.07$ & $<0.001$ \\
\hline Group A & 65 & $235.77 \pm 17.35$ & & $48.12 \pm 3.65$ & & $0.45 \pm 0.05$ & \\
\hline
\end{tabular}

outcome between two groups. The p-value of less than 0.05 was considered significant.

\section{RESULTS}

Among the 130 patients, there were 69 (53.08\%) males and $61(46.92 \%)$ females. The types of injuries were 36 $(27.69 \%)$ cases of fall from height, $44(33.85 \%)$ cases of heavy bruises, and $50(38.46 \%)$ cases of traffic injuries. According to Tile's classification, type C1 was seen in 55 $(42.31 \%)$ cases, C2 in 46 (35.38\%) cases and C3 in 29 $(22.31 \%)$ cases.

After treatment, the excellent and good rate in the group A was $96.92 \%$ (63 cases); which was higher than $81.54 \%$ (53 cases) of the group $B(p=0.005)$.

At 7 days after the operation, serum ALP level in the group A was higher than that in the group $B(p<0.001)$; serum TNF- $\alpha$ and CRP levels were lower in the group $A$ than those in the group $B$ (both $p<0.001$, Table I); serum Cor, Angll, and NE levels were lower in the group $A$ than those in the group B (all $p<0.001$, Table II).

\section{DISCUSSION}

Reconstruction plate fixation is a common surgical method for the treatment of patients with pelvic fractures. It has the advantages of complete reduction, firm fixation, and strong plasticity. However, because this treatment requires a long surgical incision in front of the pubic bone, the patient may be greatly injured. And it is not effective for unstable fractures. ${ }^{11}$ Minimally invasive percutaneous lag screw internal fixation is a clinically emerging surgical treatment in recent years. It has the advantages of small intraoperative trauma, less bleeding and short operation time.12,13 In addition, when surgical treatment is performed by internal fixation of the screw structure, the fixation is firm and the possibility of fracture or loosening after surgery is low. Therefore, its stability is good, patients can get up and exercise as soon as possible, effectively avoiding the occurrence of multiple complications. The patient can have a better physical recovery in this way. Wei et al. found treatment of unstable pelvic fractures with minimally invasive internal fixation had advantages of less bleeding, less postoperative complications, and high bone union rate. 14
In this study, group A had a better excellent and good treatment rate than the group $B$, indicating that the effect of minimally invasive percutaneous lag screw internal fixation was better than that of the reconstruction plate treatment. This conclusion was also consistent with previous researcher. ${ }^{15}$

Surgical treatment can cause greater inflammatory response of the body, coupled with the adverse stress caused by the fracture trauma; the body's inflammatory indicators will vary greatly. TNF- $\alpha$ is an inflammatory factor that changes in the early stages of inflammation and triggers and initiates inflammatory responses. It also promotes the activation and infiltration of a variety of inflammatory cells and mediates cascade activation of inflammatory responses. ${ }^{16,17} \mathrm{CRP}$ is an acute phase protein synthesised and secreted by hepatocytes when induced by proinflammatory cytokines. It has a good correlation and consistency with the progress of inflammatory reactions. ${ }^{18}$

The results of this study showed that serum TNF- $\alpha$ and CRP levels were lower in the group A than those in the group $B$ at 7 days after surgery. This showed that minimally invasive percutaneous lag screw internal fixation for unstable pelvic fractures can effectively reduce the patient's inflammatory response, reduce the damage in the body, and promote his/her early recovery. There is a close relationship between ALP and fracture healing. When the fracture is healing, osteoblasts play an extremely important role; when the bone cells are active, the number of ALPs will increase significantly, which in turn will strengthen the bone formation capacity. ${ }^{19}$ Therefore, the ability of a patient to fracture healing can be inferred by detecting serum ALP levels.

In this study, in the treatment of unstable pelvic fracture patients using minimally invasive percutaneous lag screw internal fixation, ALP level in patients was found significantly elevated higher than those of the reconstruction plate treatment, suggesting that minimally invasive percutaneous lag screw internal fixation can promote quicker healing of fractures in patients.

Cor, Angll, NE are all stress hormones. Both Cor and NE are synthesised and secreted by the adrenal glands. Cor is from the adrenal cortex and can participate in the 
regulation of glycolipids and water-sodium metabolism, exerting its permissive effect on catecholamines. NE is from the adrenal medulla and can cause vasoconstriction and increased cardiac effects. ${ }^{20}$ Ang II is a product of the activation of the renin-angiotensin-aldosterone system and has a potent vasoconstrictive activity. ${ }^{21}$

The results of this study showed that serum Cor, Ang II, and NE levels in the group A were lower than those in the group $B$ at 7 days after the operation. This showed that the minimally invasive percutaneous lag screw internal fixation could effectively reduce the surgical trauma and the degree of early postoperative stress response.

\section{CONCLUSION}

Compared with the reconstruction plate, minimally invasive percutaneous lag screw internal fixation has better clinical efficacy in patients with unstable pelvic fractures. It can effectively increase serum ALP levels, reduce the levels of inflammatory cytokines TNF- $\alpha$ and CRP, and effectively reduce surgical trauma as well as postoperative body stress response.

\section{ETHICAL APPROVAL:}

The study was approved by the Ethics Committees of the Department of orthopaedics Branch, Second People's Hospital of Liaocheng, China, prior to initiation of the research work.

\section{PATIENTS' CONSENT:}

Written informed consents on the protocol were obtained from the patients to publish the data concerning this case.

\section{CONFLICT OF INTEREST:}

Authors declared no conflict of interest.

\section{AUTHORS' CONTRIBUTION:}

JF: Written original draft and edited.

HD: Data curation and analysis.

$\mathrm{ZL}$ : Reviewed manuscript and final approval of the version to be published.

\section{REFERENCES}

1. Gandhi G, Vijayvargiya M, Shetty V, Agashe V, Maheshwari S, Monteiro J. CT-guided percutaneous sacroiliac stabilization in unstable pelvic fractures a safe and accurate technique. Rev Bras Ortop 2017; 53:323-31.

2. Shetty AP, Bosco A, Perumal R, Dheenadhayalan J, Rajasekaran S. Midterm radiologic and functional outcomes of minimallyinvasive fixation of unstable pelvic fractures using anterior internal fixator(INFIX) and percutaneous iliosacral screws. J Clin Orthop Trauma 2017; 8:241-8.

3. Yano S, Aoki Y, Watanabe A, Nakajima T, Takazawa M, Hirasawa $\mathrm{H}$, et al. Less invasive lumbopelvic fixation technique using a percutaneous pedicle screw system for unstable pelvic ring fracture in a patient with severe multiple traumas. J Neurosurg Spine 2017; 26:203-7.
4. Jazini E, Klocke N, Tannous O, Johal HS, Hao J, Salloum K, et al. Does lumbopelvic fixation add stability a cadaveric biomechanical analysis of an unstable pelvic fracture model. J Orthop Trauma 2017; 31:37-46.

5. Nascimento FP, Santili C, Akkari M, Waisberg G, Braga SR, Fucs PM. Flexible intramedullary nails with traction versus plaster cast for treating femoral shaft fractures in children comparative retrospective study. Sao Paulo Med J 2013; 131: 5-12.

6. Clasper JC, Rowley DI. Outcome following significant delays in initial surgery of ballistic femoral fractures managed without internal or external fixation. $J$ Bone Joint Surg $\mathrm{Br} 2009$; 91: 97-101.

7. Pilling E, Schneider M, Mai R, Loukota RA, Eckelt U. Minimally invasive fracture treatment with cannulated lag screws in intracapsular fractures of the condyle. $J$ Oral Maxillofac Surg 2006; 64:868-72.

8. Zhang LH, Zhang LC, Si QH, Gao Y, Su XY, Zhao Z, et al. Experimental study on treatment of acetabular anterior column fractures applyment of a minimally invasive percutaneous lag screw guide apparatus. BMC Musculoskelet Disord 2016; 17: 27-34.

9. Tempelaere C, Vincent C, Court C. Percutaneous posterior fixation for unstable pelvic ring fractures. Orthop Traumatol Surg Res 2017; 103:1169-71.

10. Chen HW, Zhao GS, Pan J. Treatment of unstable pelvic posterior ring fractures with percutaneous reconstruction plate internal fixation. China J Orthop Traumatol 2010; 23:708-10.

11. Wen Y, Liu X, Ge B, Liu Z, Shiji Z. A newer plate system for internal fixation of un-stable pelvic fractures. Int Surg 1998; 83:88-90.

12. Krieg SM, Aldinger F, Stoffel M, Meyer B, Kreutzer J. Minimally invasive decompression of chronic subdural haematomas using hollow screws efficacy and safety in a consecutive series of 320 cases. Acta Neurochir (Wien) 2012; 154:699-705.

13. Lin PP, Kang HG, Kim YI, Kim JH, Han SK. Minimally invasive surgery for femoral neck fractures using bone cement infusible hollow-perforated screw in high-risk patients with advanced cancer. Surg Oncol 2015; 24:226-31.

14. Wei $\mathrm{CH}$, Sheng ZG. Treatment of unstable pelvic fractures with minimally invasive internal fixation. China $J$ Orthop Traumatol 2010; 23:541-3.

15. Wang X, Wang G, Meng C, Liu X, Cai X. Iso-C(3D) navigation for percutaneous sacroiliac joint screw internal fixation for treatment of pelvic posterior ring injuries. Chin J Rep Reconstr Surg 2016; 30:1338-43.

16. Yeo E S, Hwang JY, Park JE, Choi YJ, Huh KB, Kim WY. Tumor necrosis factor (TNF- $\alpha$ ) and C-reactive protein (CRP) are positively associated with the risk of chronic kidney disease in patients with type 2 diabetes. Yonsei Med J 2010; 51:519-25.

17. Gokulakrishnan K, Deepa R, Mohan V. Association of high sensitivity C-reactive protein (hsCRP) and tumour necrosis factor-alpha (TNF- $\alpha$ ) with carotid intimal medial thickness in subjects with different grades of glucose intolerance the chennai urban rural epidemiology study (CURES-31). Clin Biochem 2008; 41:480-5.

18. Bahceci M, Gokalp D, Bahceci S, Tuzcu A, Atmaca S, Arikan S. The correlation between adiposity and adiponectin, tumor 
necrosis factor alpha, interleukin-6 and high sensitivity C-reactive protein levels. Is adipocyte size associated with inflammation in adults. J Endocrinol Invest 2007; 30:210-4.

19. Muljaci A, Poljakguberina R, Zivkovi O, Bili V, Guberina M, Crvenkovi $D$. Course and rate of post-fracture bone healing in correlation with bone-specific alkaline phosphatase and bone callus formation. Coll Antropol 2013; 37:1275-83.
20. Sasaoka T, Egi Y, Tawa M, Yamamoto A, Ohkita M, Takaoka M, et al. Angiotensin type 2 receptor-mediated inhibition of norepinephrine release in isolated rat hearts. J Cardiovasc Pharmacol 2008; 52:176-83.

21. Wen H, Gwathmey JK, Xie LH. Oxidative stress-mediated effects of angiotensin in the cardiovascular system. World $\mathrm{J}$ Hypertens 2012; 2:34-44.

.......... 